\title{
THE HIJAZI INEQUALITY ON MANIFOLDS WITH BOUNDARY
}

\author{
SIMON RAULOT
}

\begin{abstract}
In this paper, we prove the Hijazi inequality on compact Riemannian spin manifolds under two boundary conditions: the condition associated with a chirality operator and the Riemannian version of the MIT bag condition. We then show that the limiting-case is characterized as being a half-sphere for the first condition whereas the equality cannot be achieved for the second.
\end{abstract}

\section{INTRODUCTION}

In [Fri80], T. Friedrich established an inequality relating the eigenvalues of the Dirac operator on a compact $n$-dimensional Riemannian spin manifold without boundary with its scalar curvature R. This inequality is given by

$$
\lambda^{2} \geq \frac{n}{4(n-1)} \mathrm{R}_{0}
$$

where $\mathrm{R}_{0}$ denotes the infimum of the scalar curvature of $\mathrm{M}$. For $n \geq 3$, using the conformal covariance of the Dirac operator O. Hijazi Hij86 proved that

$$
\lambda^{2} \geq \frac{n}{4(n-1)} \mu_{1}(\mathrm{~L})
$$

where $\mu_{1}(\mathrm{~L})$ is the first eigenvalue of the conformal Laplacian given by $\mathrm{L}=\frac{4(n-1)}{n-2} \Delta+R$. The operator $\mathrm{L}$ is a second order conformally covariant differential operator relating the scalar curvatures of two metrics in the same conformal class. In Hij91, O. Hijazi derives a conformal lower bound for any eigenvalues $\lambda$ of the Dirac operator involving the Yamabe invariant $\mu(\mathrm{M})$. Indeed, he proved that if $n \geq 3$, then

$$
\lambda^{2} \operatorname{vol}(\mathrm{M}, g)^{\frac{2}{n}} \geq \frac{n}{4(n-1)} \mu(\mathrm{M}) .
$$

The Yamabe invariant $\mu(\mathrm{M})$ has been introduced in Yam60 in order to solve the following problem now called the Yamabe problem: given a closed compact Riemannian manifold $\left(\mathrm{M}^{n}, g\right)$, is there a metric in the conformal class of $g$ such that the scalar curvature is constant? For $n=2$, C. Bär Bär92 showed that

$$
\lambda^{2} \geq \frac{2 \pi \chi\left(\mathrm{M}^{2}\right)}{\operatorname{Area}\left(\mathrm{M}^{2}, g\right)},
$$

Date: June 20, 2021.

1991 Mathematics Subject Classification. Differential Geometry, Global Analysis, 53C27, 53C40, $53 \mathrm{C} 80,58 \mathrm{G} 25$.

Key words and phrases. Manifolds with Boundary, Dirac Operator, Conformal Geometry, Spectrum, Yamabe Problem. 
where $\chi\left(\mathrm{M}^{2}\right)$ is the Euler characteristic class. A natural question is then to ask if those results still hold if we consider manifolds with boundary. In [HMR02, the authors prove Friedrich-type inequalities under four elliptic boundary conditions and under some curvature assumptions (the non-negativity of the mean curvature). Two of these boundary conditions are (global) Atiyah-Patodi-Singer type conditions and two local boundary conditions. The present paper being devoted to the conformal aspect of those results, the choice of the boundary condition is important. As pointed out in HMZ02, the AtiyahPatodi-Singer type conditions are not conformally invariant, while the local boundary conditions are indeed conformally invariant. Moreover we don't assume that the boundary $\partial \mathrm{M}$ has nonnegative mean curvature and then we prove:

Theorem 1. Let $\left(\mathrm{M}^{n}, g\right)$ be an $n$-dimensional connected compact Riemannian spin manifold with non-empty boundary $\partial \mathrm{M}$. For $n \geq 3$ if $\mathrm{M}$ has positive Yamabe invariant and for $n=2$ if $\mathrm{M}$ is a surface of genus 0 whith compact connected boundary, then:

1) Under the CHI boundary condition associated with a chirality operator (see Section (4), the spectrum of the Dirac operator $\mathrm{D}$ of $\mathrm{M}$ is a sequence of unbounded real numbers $\left\{\lambda_{k}^{\mathrm{CHI}} / k \in \mathbb{Z}\right\}$. For $n \geq 3$, any eigenvalue $\lambda^{\mathrm{CHI}}$ of the Dirac operator satisfies

$$
\left(\lambda^{\mathrm{CHI}}\right)^{2} \geq \frac{n}{4(n-1)} \mu_{1}(\mathrm{~L}) .
$$

For $n=2$, we have

$$
\left(\lambda^{\mathrm{CHI}}\right)^{2} \geq \frac{2 \pi}{\operatorname{Area}\left(\mathrm{M}^{2}, g\right)} .
$$

Moreover, equality holds if and only if the manifold $\mathrm{M}$ is isometric to the halfsphere $\mathbb{S}_{+}^{n}(r)$ with radius $r$, where $r$ depends on the first eigenvalue of the Dirac operator under this boundary condition.

2) Under the MIT boundary condition (see Remark 3), the spectrum of the Dirac operator $\mathrm{D}$ is an unbounded discrete set of complex numbers $\left\{\lambda_{k}^{\mathrm{MIT}} / k \in \mathbb{Z}\right\}$ with positive imaginary part. For $n \geq 3$, any eigenvalue $\lambda^{\mathrm{MIT}}$ of the Dirac operator satisfies

$$
\left|\lambda^{\mathrm{MIT}}\right|^{2}>\frac{n}{4(n-1)} \mu_{1}(\mathrm{~L})
$$

For $n=2$, we have

$$
\left|\lambda^{\mathrm{MIT}}\right|^{2}>\frac{2 \pi}{\operatorname{Area}\left(\mathrm{M}^{2}, g\right)} .
$$

The real number $\mu_{1}(\mathrm{~L})$ is the first eigenvalue of the eigenvalue boundary problem

$$
\begin{cases}\mathrm{L} u=\mu_{1}(\mathrm{~L}) u & \text { on } \mathrm{M} \\ \mathrm{B} u=0 & \text { along } \partial \mathrm{M}\end{cases}
$$

with $\mathrm{B}$ the mean curvature operator acting on smooth fonctions on the manifold $\mathrm{M}$.

Finally, we extend Inequality (3) to the case of manifolds with boundary. The author would like to thank the referee for helpful comments. 


\section{Spin Manifolds With Boundary}

In this section, we summarize some basic facts about spin manifolds with boundary. Standard references on this subject can be found in [HMZ02. Let $\left(\mathrm{M}^{n}, g\right)$ be an $n$ dimensional Riemannian spin manifold with boundary and denote by $\nabla$ the Levi-Civita connection on the tangent bundle TM and the associated bundles. We choose a spin structure and denote by $\operatorname{Spin}(\mathrm{M})$ the principal bundle with structural group $\operatorname{Spin}_{n}$ given by this spin structure. The spinor bundle on the manifold $M$ is then the complex vector bundle of rank $2^{\left[\frac{n}{2}\right]}$, denoted by $\Sigma \mathrm{M}$, associated with the complex spinor representation. This representation provides a Clifford multiplication

$$
\gamma: \mathbb{C l}(\mathrm{M}) \longrightarrow \operatorname{End}(\Sigma \mathrm{M})
$$

which is a fibre preserving algebra morphism. The spinor bundle $\Sigma \mathrm{M}$ is endowed with a natural hermitian product, denoted by $\langle$,$\rangle , and with a spinorial Levi-Civita connection$ $\nabla$ acting on spinor fields, i.e. on sections of the spinor bundle (see [LM89] or [Fri00], for example). We can easily show that the spinorial connection $\nabla$ is compatible with the hermitian product $\langle$, $\rangle$, i.e.

$$
X\langle\psi, \varphi\rangle=\left\langle\nabla_{X} \psi, \varphi\right\rangle+\left\langle\psi, \nabla_{X} \varphi\right\rangle,
$$

for all $\psi, \varphi \in \Gamma(\Sigma \mathrm{M})$ and for all $X \in \Gamma(\mathrm{TM})$. Moreover, they also satisfy the following properties:

$$
\begin{array}{r}
\langle\gamma(X) \psi, \gamma(X) \varphi\rangle=g(X, X)\langle\psi, \varphi\rangle \\
\nabla_{X}(\gamma(Y) \psi)=\gamma\left(\nabla_{X} Y\right) \psi+\gamma(Y) \nabla_{X} \psi,
\end{array}
$$

for all $\psi, \varphi \in \Gamma(\Sigma \mathrm{M})$ and for all $X, Y \in \Gamma(\mathrm{TM})$. Note that Identity (6) implies that Clifford multiplication by a unit tangent vector field is skew-symmetric on $\Sigma \mathrm{M}$. The Dirac operator D on $\Sigma \mathrm{M}$ is then the first order elliptic operator locally given by

$$
\begin{aligned}
\mathrm{D}: \Gamma(\Sigma \mathrm{M}) & \longrightarrow \Gamma\left(\sum \mathrm{M}\right) \\
\psi & \longmapsto \sum_{i=1}^{n} \gamma\left(e_{i}\right) \nabla_{e_{i}} \psi,
\end{aligned}
$$

where $\left\{e_{1}, \ldots, e_{n}\right\}$ is a local orthonormal frame of TM.

Consider now the boundary $\partial \mathrm{M}$ which is an oriented Riemannian hypersurface of $\mathrm{M}$ with induced orientation and Riemannian structure. Then there exists a unit vector field $\nu$ normal to the boundary which allows to pull-back the spin structure over $\mathrm{M}$ to a spin structure over the boundary $\partial \mathrm{M}$. Hence we have that the restriction

$$
\mathbf{S}(\partial \mathrm{M}):=\Sigma \mathrm{M}_{\mid \partial \mathrm{M}}
$$

is a left module over $\mathbb{C l}(\partial \mathrm{M})$ with Clifford multiplication

$$
\gamma^{\mathbf{S}}: \mathbb{C} l(\partial \mathrm{M}) \longrightarrow \operatorname{End}(\mathbf{S}(\partial \mathrm{M}))
$$

given by $\gamma^{\mathbf{S}}(X) \psi=\gamma(X) \gamma(\nu) \psi$ for all $X \in \Gamma(\mathrm{TM})$ and $\psi \in \Gamma(\mathbf{S}(\partial \mathrm{M}))$. Now let $\nabla^{\partial \mathrm{M}}$ be the Levi-Civita connection of the boundary $(\partial \mathrm{M}, g)$ and let $\left(e_{1}, \ldots, e_{n-1}, e_{n}=\nu\right)$ be a local orthonormal frame of TM, then the Riemannian Gauss formula states that for $1 \leq i, j \leq n-1$,

$$
\nabla_{e_{i}} e_{j}=\nabla_{e_{i}}^{\partial \mathrm{M}} e_{j}+g\left(A e_{i}, e_{j}\right) \nu
$$


where $A X=-\nabla_{X} \nu$ is the shape operator of the boundary $\partial \mathrm{M}$. We can then relate the two associated spin connections. Indeed, if $\nabla$ (resp. $\left.\nabla^{\mathbf{S}}\right)$ is the Levi-Civita connection on the spinor bundle $\Sigma \mathrm{M}$ (resp. $\mathbf{S}(\partial \mathrm{M})$ ), we have the spinorial Gauss Formula (for more details, see [Tra95], Bär98] or [Mor01]):

$$
\left(\nabla_{X} \psi\right)_{\mid \partial \mathrm{M}}=\nabla_{X}^{\mathbf{S}} \psi_{\mid \partial \mathrm{M}}+\frac{1}{2} \gamma^{\mathbf{S}}(A X) \psi_{\mid \partial \mathrm{M}}
$$

for all $X \in \Gamma(\mathrm{T}(\partial \mathrm{M}))$ and for all $\psi \in \Gamma(\Sigma \mathrm{M})$. The spinor bundle $\mathbf{S}(\partial \mathrm{M})$ is also endowed with a Hermitian metric denoted by $\langle$,$\rangle induced from that on \Sigma \mathrm{M}$. The induced metric, the Clifford multiplication and the Levi-Civita connection satisfy properties (5), (6) and (17) , i.e. the spinor bundle $\mathbf{S}(\partial \mathrm{M})$ is a Dirac bundle.

The induced spin structure on the boundary allows to construct an intrinsic spinor bundle $\Sigma(\partial \mathrm{M})$ over $\partial \mathrm{M}$. This bundle is naturally endowed with a Hermitian metric, a Clifford multiplication $\gamma^{\partial \mathrm{M}}$ and a spinorial Levi-Civita connection $\nabla^{\partial \mathrm{M}}$. It is not difficult to show that this bundle can be identified with the restricted spinor bundle $\mathbf{S}(\partial \mathrm{M})$ if $n$ is odd. In this case, the Clifford multiplication and the Levi-Civita connection on $\Sigma(\partial \mathrm{M})$ correspond to the Clifford multiplication and the Levi-Civita connection on $\mathbf{S}(\partial \mathrm{M})$. If $n$ is even, the spinor bundle $\mathbf{S}(\partial \mathrm{M})$ could be identified with the direct sum $\Sigma(\partial \mathrm{M}) \oplus \Sigma(\partial \mathrm{M})$. Moreover, the Clifford multiplication $\gamma^{\mathbf{S}}$ correspond to $\gamma^{\partial \mathrm{M}} \oplus-\gamma^{\partial \mathrm{M}}$ and the Levi-Civita connection $\nabla^{\mathrm{S}}$ to $\nabla^{\partial \mathrm{M}} \oplus \nabla^{\partial \mathrm{M}}$.

We can now define several Dirac operators acting on sections of these bundles (for a complete review of these operators, see [Mor01]). However, in our case, the most important one is the boundary Dirac operator acting on sections of $\mathbf{S}(\partial \mathrm{M})$. This operator is given by composition of the Clifford multiplication $\gamma^{\mathbf{S}}$ and the spinorial Levi-Civita connection $\nabla^{\mathbf{S}}$. This operator is denoted by $\mathrm{D}^{\mathbf{S}}$ and is locally given by

$$
\mathrm{D}^{\mathbf{S}}(\psi)=\sum_{i=1}^{n-1} \gamma^{\mathbf{S}}\left(e_{i}\right) \nabla_{e_{i}}^{\mathbf{S}} \psi .
$$

Note that in the case of a closed compact manifold without boundary, the classical Dirac operator has exactly one self-adjoint $\mathrm{L}^{2}$ extention, so it has real discrete spectrum. In the case of a manifold with boundary, a defect of symmetry appears, given by the formula

$$
\int_{\mathrm{M}}\langle\mathrm{D} \psi, \varphi\rangle d v(g)-\int_{\mathrm{M}}\langle\psi, \mathrm{D} \varphi\rangle d v(g)=-\int_{\partial \mathrm{M}}\langle\gamma(\nu) \psi, \varphi\rangle d s(g),
$$

for $\psi, \varphi \in \Gamma(\Sigma \mathrm{M})$, and where $\nu$ is the inner unit vector field along the boundary and $d v(g)$ (resp. $d s(g)$ ) is the volume form of the manifold $\mathrm{M}$ (resp. the boundary $\partial \mathrm{M}$ ). According to this formula, we note that the Dirac operator D is not symmetric, but we will see that, under suitable boundary conditions, the l.h.s of (8) vanishes.

In order to prove Theorem 11 which is an estimate of the fundamental Dirac operator eigenvalues of the ambient manifold $\mathrm{M}$ under suitable boundary conditions, we will give an inequality called "spinorial Reilly inequality" relating the geometry of the manifold M and that of its boundary $\partial \mathrm{M}$ (see [HMR02, [HMZ01 or [HMZ02]). The spinorial Reilly inequality is based on the Schrödinger-Lichnerowicz formula, given by

$$
\mathrm{D}^{2}=\nabla^{*} \nabla+\frac{1}{4} \mathrm{R},
$$


where $\mathrm{R}$ is the scalar curvature of $\mathrm{M}$. An integral version of this formula leads to (see HMR02 for a proof of the following proposition):

Proposition 2. For all spinor fields $\psi \in \Gamma(\Sigma \mathrm{M})$, we have:

$$
\int_{\partial \mathrm{M}}\left(\left\langle\mathrm{D}^{\mathrm{S}} \psi, \psi\right\rangle-\frac{n-1}{2} \mathrm{H}|\psi|^{2}\right) d s(g) \geq \int_{\mathrm{M}}\left(\frac{1}{4} \mathrm{R}|\psi|^{2}-\frac{n-1}{n}|\mathrm{D} \psi|^{2}\right) d v(g),
$$

where $\mathrm{R}$ is the scalar curvature of the manifold $\mathrm{M}, \mathrm{H}=\frac{1}{n-1} \operatorname{tr}(A)$ is the mean curvature of the boundary and $d v(g)$ (resp. $d s(g)$ ) is the Riemannian volume form of $\mathrm{M}$ (resp. $\partial \mathrm{M}$ ). Moreover equality occurs if the spinor field $\psi$ is a twistor-spinor, i.e. if it satisfies $\mathrm{P} \psi=0$ where $\mathrm{P}$ is the twistor operator acting on $\Sigma \mathrm{M}$ which is locally given for all $X \in \Gamma(\mathrm{TM})$ by:

$$
\mathrm{P}_{X} \psi=\nabla_{X} \psi+\frac{1}{n} \gamma(X) \mathrm{D} \psi
$$

The proof of Theorem 1 is based on the conformal covariance of the fundamental Dirac operator D of the manifold M; we now summarize some classical facts about Dirac operators in a conformal class of the Riemannian metric $g$. So consider a nowhere vanishing function $h$ on the manifold $\mathrm{M}$, and let $\bar{g}=h^{2} g$ be a conformal change of the metric. Then we have an obvious identification between the two $\mathrm{SO}_{n}$-principal bundles of $g$ and $\bar{g}$-orthonormal oriented frames denoted respectively by $\mathrm{SO}(\mathrm{M})$ and $\mathrm{SO}(\overline{\mathrm{M}})$. We can thus identify the corresponding $\operatorname{Spin}_{n}$-principal bundles $\operatorname{Spin}(\mathrm{M})$ and $\operatorname{Spin}(\overline{\mathrm{M}})$ and this leads to a bundle isometry

$$
\begin{array}{ccc}
\Sigma \mathrm{M} & \longrightarrow \Sigma \overline{\mathrm{M}} \\
\varphi & \longmapsto \bar{\varphi} .
\end{array}
$$

For more details, we refer to Hit74, Hij86 or Bau89. We can also relate the corresponding Levi-Civita connections and Clifford multiplications. Indeed, denoting by $\bar{\nabla}$ and $\bar{\gamma}$ the associated data which act on the bundle $\Sigma \overline{\mathrm{M}}$. We can easily show that

$$
\bar{\gamma}=h \gamma, \quad \bar{\nabla}_{X} \psi-\nabla_{X} \psi=-\frac{1}{2 h} \gamma(X) \gamma(\nabla h) \psi-\frac{1}{2 h} g(\nabla h, X) \psi
$$

A result due to Hitchin [Hit74] gives the conformal covariance of the Dirac operator:

Proposition 3. Let D (resp. $\overline{\mathrm{D}}$ ) be the fundamental Dirac operator on the manifold $\left(\mathrm{M}^{n}, g\right)$ (resp. $\left.\left(\mathrm{M}^{n}, \bar{g}\right)\right)$, then we have the following identity:

$$
\overline{\mathrm{D}}(\bar{\psi})=h^{-\frac{n+1}{2}} \overline{\mathrm{D}\left(h^{\frac{n-1}{2}} \psi\right)}
$$

for all $\psi \in \Gamma(\Sigma M)$.

It is an obvious fact that this conformal change of the metric induces a conformal change of the metric on the boundary. We can then identify the connections and the Clifford multiplications of $\mathbf{S}(\partial \mathrm{M})$ and $\mathbf{S}(\overline{\partial \mathrm{M}})$. In the same way, the boundary Dirac operators $\mathrm{D}^{\mathbf{S}}$ and $\overline{\mathrm{D}} \mathbf{S}$, acting respectively on $\mathbf{S}(\partial \mathrm{M})$ and $\mathbf{S}(\overline{\partial \mathrm{M}})$, satisfy

$$
\overline{\mathrm{D}}^{\mathbf{S}}(\bar{\psi})=h^{-\frac{n}{2}} \overline{\mathrm{D}^{\mathbf{S}}\left(h^{\frac{n-2}{2}} \psi\right)},
$$

for all $\psi \in \Gamma(\mathbf{S}(\partial \mathrm{M}))$. For more details on these identifications, we refer to [HMZ02. 


\section{Local Elliptic Boundary CONDitions for the DiRAC operator}

In order to prove Theorem 1, we have to use suitable boundary conditions for the fundamental Dirac operator D on the manifold M. In other words, we look for conditions

$$
\mathbb{B}: \mathrm{L}^{2}(\mathrm{~S}(\partial \mathrm{M})) \longrightarrow \mathrm{L}^{2}(\mathrm{~V})
$$

where $\mathrm{V}$ is a Hermitian vector bundle over the boundary $\partial \mathrm{M}$, to impose on the restrictions of spinor fields on $\mathrm{M}$ to the boundary $\partial \mathrm{M}$ such that the Dirac operator is a Fredholm operator, i.e. for given data $\Phi \in \Gamma(\Sigma \mathrm{M})$ and $\chi \in \Gamma(\mathrm{V})$ the following boundary value problem

$$
\begin{cases}\mathrm{D} \psi=\Phi & \text { on } \mathrm{M} \\ \mathbb{B}\left(\psi_{\mid \partial \mathrm{M}}\right)=\chi & \text { along } \partial \mathrm{M}, \quad(B P)\end{cases}
$$

has a unique solution up to a finite dimensional kernel. Moreover the following eigenvalue problem

$$
\begin{cases}\mathrm{D} \varphi=\lambda \varphi & \text { on } \mathrm{M} \\ \mathbb{B}\left(\varphi_{\mid \partial \mathrm{M}}\right)=0 & \text { along } \partial \mathrm{M}\end{cases}
$$

should have a discrete spectrum with finite dimensional eigenspaces, unless it is the whole complex plane. The preceding properties are satisfied if the operator $\mathbb{B}$ satisfies some ellipticity conditions. We follow HMR02 for the notion of ellipticity of a boundary condition for the Dirac operator (for more general cases, we refer to [Hör85] and [Lop53). In fact, the principal tool for finding well-posed conditions was discovered by Caldéron and is called the Caldéron projector of the Dirac operator $\mathrm{D}$, denoted by $\mathcal{P}_{+}(\mathrm{D})$. This projector is a pseudo-differential operator of order zero which has the particularity that its principal symbol $\sigma\left(\mathcal{P}_{+}(\mathrm{D})\right)$ detects ellipticity, i.e. such that problems $(B P)$ and $(E B P)$ could be solved. Indeed, in BBW93, the authors show that a pseudo-differential operator

$$
\mathbb{B}: \mathrm{L}^{2}\left(\Sigma \mathrm{M}_{\mid \partial \mathrm{M}}\right) \longrightarrow \mathrm{L}^{2}(\mathrm{~V})
$$

defines an elliptic boundary condition for the operator D if it satisfies the following property:

$$
\sigma(\mathbb{B})(u)_{\mid \operatorname{Im} \sigma\left(\mathcal{P}_{+}(\mathrm{D})\right)(u)}: \operatorname{Im} \sigma\left(\mathcal{P}_{+}(\mathrm{D})\right)(u) \subset \Sigma_{p} \mathrm{M} \longrightarrow \mathrm{V}_{p}
$$

is an isomorphism on its image for all nontrivial $u \in \mathrm{T}_{p}(\partial \mathrm{M})$ and all $p \in \partial \mathrm{M}$. Moreover if the rank of the vector bundle $\mathrm{V}$ and the dimension of $\operatorname{Im} \sigma\left(\mathcal{P}_{+}(\mathrm{D})\right)$ are the same then the boundary condition is said to be local. As the principal symbol of the Caldéron projector of the Dirac operator is given by (see BBW93])

$$
\sigma\left(\mathcal{P}_{+}(\mathrm{D})\right)(u)=\frac{1}{2|u|}\left(i \gamma^{\mathbf{S}}(u)+|u| \mathrm{Id}\right)
$$

for each nontrivial $u \in \mathrm{T}_{p}(\partial \mathrm{M})$ and $p \in \partial \mathrm{M}$, we obtain the following result which gives ellipticity of a boundary condition for the Dirac operator D on a manifold with boundary (see [HMR02]):

Proposition 4. Let $\left(\mathrm{M}^{n}, g\right)$ be an $n$-dimensional compact Riemannian spin manifold with non-empty boundary $\partial \mathrm{M}$. Then, a pseudo-differential operator

$$
\mathbb{B}: \mathrm{L}^{2}(\mathbf{S}(\partial \mathrm{M})) \longrightarrow \mathrm{L}^{2}(\mathrm{~V}),
$$


where $\mathrm{V} \rightarrow \partial \mathrm{M}$ is a Hermitian vector bundle, is an elliptic boundary condition for the Dirac operator $\mathrm{D}$ of $\mathrm{M}$ if and only if its principal symbol

$$
\sigma(\mathbb{B}): \mathrm{T}(\partial \mathrm{M}) \longrightarrow \operatorname{Hom}_{\mathbb{C}}(\mathbf{S}(\partial \mathrm{M}), \mathrm{V})
$$

satisfies the following two conditions

(1) $\operatorname{Ker} \sigma(\mathbb{B})(u) \cap\left\{\varphi \in \Sigma_{p} \mathrm{M} / i \gamma(\nu) \gamma(u) \varphi=-|u| \varphi\right\}=\{0\}$,

(2) $\operatorname{dim} \operatorname{Im} \sigma(\mathbb{B})(u)=\frac{1}{2} \operatorname{dim} \Sigma_{p} \mathrm{M}=2^{\left[\frac{n}{2}\right]-1}$.

Moreover, if $\mathrm{V}$ is a bundle with rank $\frac{1}{2} \operatorname{dim} \Sigma_{p} \mathrm{M}=2^{\left[\frac{n}{2}\right]-1}$, we have a local elliptic boundary condition. When these ellipticity conditions are satisfied, the following eigenvalue boundary problem

$$
\begin{cases}\mathrm{D} \psi=\lambda \psi & \text { on } \mathrm{M} \\ \mathbb{B}\left(\psi_{\mid \partial \mathrm{M}}\right)=0 & \text { along } \partial \mathrm{M}\end{cases}
$$

has a discrete spectrum with finite dimensional eigenspaces consisting of smooth spinor fields, unless it is the whole complex plane.

We are now ready to study elliptic boundary conditions for the Dirac operator.

\section{The CONDITION ASSOCIATED WITH A CHIRALITY OPERATOR}

In this section, we consider an $n$-dimensional compact Riemannian spin manifold $\left(\mathrm{M}^{n}, g\right)$ with non-empty boundary equipped with a chirality operator. First recall the definition of such an operator. A linear map

$$
\mathrm{G}: \Gamma(\Sigma \mathrm{M}) \longrightarrow \Gamma(\Sigma \mathrm{M})
$$

is a chirality operator if it satisfies the following properties:

$$
\begin{array}{r}
\mathrm{G}^{2}=\mathrm{Id}, \quad\langle\mathrm{G} \varphi, \mathrm{G} \psi\rangle=\langle\varphi, \psi\rangle \\
\nabla_{X}(\mathrm{G} \psi)=\mathrm{G}\left(\nabla_{X} \psi\right), \quad \gamma(X) \mathrm{G} \psi=-\mathrm{G}(\gamma(X) \psi)
\end{array}
$$

for all $\varphi, \psi \in \Gamma(\Sigma \mathrm{M})$ and $X \in \Gamma(\mathrm{TM})$. Note that such an operator does not exist on all manifolds. However, we can note that if the dimension $n=2 m$ of the manifold $\mathrm{M}$ is even, then $\mathrm{G}=\gamma\left(\omega_{2 m}\right)$, where $\omega_{2 m}$ is the volume element of the spinor bundle, is a chirality operator. Now consider the fiber preserving endomorphism

$$
\gamma(\nu) \mathrm{G}: \Gamma(\mathbf{S}(\partial \mathrm{M})) \longrightarrow \Gamma(\mathbf{S}(\partial \mathrm{M}))
$$

acting on the restriction of the spinor bundle $\Sigma \mathrm{M}$ to the boundary. We can easily check that this map is pointwise self-adjoint and is involutive. So the bundle $\mathbf{S}(\partial \mathrm{M})$ can be decomposed into two eigensubbundles $\mathrm{V}^{ \pm}$associated with the eigenvalues \pm 1 . Now we can define the two pointwise orthogonal projections:

$$
\begin{aligned}
\mathbb{B}_{\mathrm{CHI}}^{ \pm}: \mathrm{L}^{2}(\mathrm{~S}(\partial \mathrm{M})) & \longrightarrow \mathrm{L}^{2}\left(\mathrm{~V}^{ \pm}\right) \\
\varphi & \longmapsto \frac{1}{2}(\mathrm{Id} \pm \gamma(\nu) \mathrm{G}) \varphi,
\end{aligned}
$$

and it is easy to check that these operators satisfy the ellipticity conditions given in Proposition 4 (see HMR02]). 
Remark 1. It is an important fact to note that a chirality operator $\mathrm{G}$ acting on sections of $\Sigma \mathrm{M}$ allows to construct a chirality operator $\overline{\mathrm{G}}$ acting on $\Sigma \overline{\mathrm{M}}$. Indeed, the operator defined by

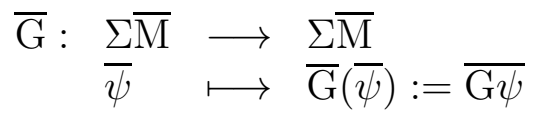

is a chirality operator acting on $\Sigma \overline{\mathrm{M}}$.

We can now prove the first part of the Theorem [

Theorem 5. Let $\left(\mathrm{M}^{n}, g\right)$ be an n-dimensional compact Riemannian spin manifold with non-empty boundary $\partial \mathrm{M}$ and with positive Yamabe invariant. Under the $\mathrm{CHI}$ boundary condition, the spectrum of the Dirac operator $\mathrm{D}$ of $\mathrm{M}$ is a non-decreasing sequence of real numbers $\left\{\lambda_{k}^{\mathrm{CHI}} / k \in \mathbb{Z}\right\}$ which satisfies

$$
\left(\lambda_{k}^{\mathrm{CHI}}\right)^{2} \geq \frac{n}{4(n-1)} \mu_{1}(\mathrm{~L}) .
$$

Moreover, equality holds if and only if $\mathrm{M}$ is conformally equivalent to the half-sphere $\mathbb{S}_{+}^{n}(r)$, where $r$ depends of the first eigenvalue of $\mathrm{D}$.

Proof: The spectrum is real because under this boundary condition, the Dirac operator is symmetric. Indeed, if $\varphi$ and $\psi$ satisfy $\mathbb{B}_{\mathrm{CHI}}^{ \pm}\left(\varphi_{\mid \partial \mathrm{M}}\right)=\mathbb{B}_{\mathrm{CHI}}^{ \pm}\left(\psi_{\mid \partial \mathrm{M}}\right)=0$, then we have:

$$
\langle\gamma(\nu) \psi, \varphi\rangle=\langle\mathrm{G}(\gamma(\nu) \psi), \mathrm{G} \varphi\rangle=-\langle\gamma(\nu) \psi, \varphi\rangle,
$$

hence by Formula (8), the symmetry property follows by integration. In fact, we can show that under this boundary condition, the Dirac operator extends to a self-adjoint linear operator on $\mathrm{L}^{2}$ (see [DW95] for this boundary condition or [Hör85] for a more general case). Furthermore, we have seen in Section 3 that the eigenvalue boundary problem

$$
\begin{cases}\mathrm{D} \psi=\lambda_{k}^{\mathrm{CHI}} \psi & \text { on } \mathrm{M} \\ \mathbb{B}_{\mathrm{CHI}}^{ \pm}\left(\psi_{\mid \partial \mathrm{M}}\right)=0 & \text { along } \partial \mathrm{M},\end{cases}
$$

admits a smooth solution $\psi \in \Gamma(\Sigma \mathrm{M})$ because of the ellipticity of the CHI boundary condition. Let $\bar{g}=f^{\frac{4}{n-2}} g$ be a conformal change of the metric and consider the spinor field $\varphi=f^{-\frac{n-1}{n-2}} \psi \in \Gamma(\Sigma \mathrm{M})$. Using the conformal covariance of the Dirac operator given in Proposition 3, the spinor field $\bar{\varphi} \in \Gamma(\Sigma \overline{\mathrm{M}})$ satisfies

$$
\overline{\mathrm{D}}(\bar{\varphi})=\lambda_{k}^{\mathrm{CHI}} f^{-\frac{2}{n-2}} \bar{\varphi}
$$

Now putting this spinor field in the spinorial Reilly inequality (9) expressed in the metric $\bar{g}$ gives

$$
\begin{aligned}
\int_{\mathrm{M}}\left(\frac{1}{4} \overline{\mathrm{R}}|\bar{\varphi}|^{2}-\frac{n-1}{n}\left|\lambda_{k}^{\mathrm{CHI}}\right|^{2} f^{-\frac{4}{n-2}}|\bar{\varphi}|^{2}\right) d v(\bar{g}) \\
\leq \int_{\partial \mathrm{M}}\left(\left\langle\overline{\mathrm{D}}^{\mathbf{S}}(\bar{\varphi}), \bar{\varphi}\right\rangle-\frac{n-1}{2} \overline{\mathrm{H}}|\bar{\varphi}|^{2}\right) d s(\bar{g}),
\end{aligned}
$$


where $\overline{\mathrm{R}}$ (resp. $\overline{\mathrm{H}}$ ) is the scalar curvature (resp. the mean curvature) of the manifold $\left(\mathrm{M}^{n}, \bar{g}\right)$ (resp. of the boundary $(\partial \mathrm{M}, \bar{g})$ ). However for $n \geq 3$, we can express the corresponding curvatures using the conformal Laplacian

$$
\mathrm{L} u=\frac{4(n-1)}{n-2} \Delta u+\mathrm{R} u
$$

and the conformal mean curvature operator

$$
\mathrm{B} u=\frac{2}{n-2} \frac{\partial u}{\partial \nu}+\mathrm{H} u
$$

Indeed, we have

$$
\overline{\mathrm{R}}=f^{-\frac{n+2}{n-2}} \mathrm{~L} f, \quad \overline{\mathrm{H}}=f^{-\frac{n}{n-2}} \mathrm{~B} f,
$$

where the function $f$ is the conformal factor of the metric $\bar{g}$. Now recall that the eigenvalue boundary problem

$$
\begin{cases}\mathrm{L} u=\mu_{1}(\mathrm{~L}) u & \text { on } \mathrm{M} \\ \mathrm{B} u=0 & \text { along } \partial \mathrm{M},\end{cases}
$$

appearing in the statement of this theorem, was introduced by Escobar in Esc92 in the context of the Yamabe problem for manifolds with boundary. He proved that the sign of the corresponding first eigenvalue $\mu_{1}(\mathrm{~L})$, whose variational characterization is given by

$$
\mu_{1}(\mathrm{~L})=\inf _{u \in C^{1}(\mathrm{M}), u \neq 0} \frac{\int_{\mathrm{M}}\left(\frac{2}{n-2}|\nabla u|^{2}+\frac{1}{2(n-1)} \mathrm{R} u^{2}\right) d v(g)+\int_{\partial \mathrm{M}} \mathrm{H} u^{2} d s(g)}{\int_{\mathrm{M}} u^{2} d s(g)},
$$

is invariant under conformal change of the metric on $\mathrm{M}$ and that an associated eigenfunction $f$ has to be positive. Moreover, Escobar showed that $\mu_{1}(\mathrm{~L})$ has to be positive (resp. zero or negative) if and only if there exists a conformally related metric on $\mathrm{M}$ with positive (resp. zero or negative) scalar curvature and such that the boundary is minimal. Now choosing the conformal factor of $\bar{g}$ to be a positive eigenfunction $f_{1}$ associated with $\mu_{1}(\mathrm{~L})$ in Inequality (18) and using the relations (19) lead to

$$
\int_{\mathrm{M}}\left(\frac{1}{4} \mu_{1}(\mathrm{~L})-\frac{n-1}{n}\left|\lambda^{\mathrm{CHI}}\right|^{2}\right)|\bar{\varphi}|^{2} f_{1}^{-\frac{4}{n-2}} d v(\bar{g}) \leq \int_{\partial \mathrm{M}}\left\langle\overline{\mathrm{D}}^{\mathbf{S}}(\bar{\varphi}), \bar{\varphi}\right\rangle d s(\bar{g}) .
$$

We now prove that the boundary term vanishes under the CHI boundary condition. Indeed, using the conformal covariance of the boundary Dirac operator given in (14), we have

$$
\overline{\mathrm{D}} \mathbf{S}(\bar{\varphi})=f_{1}^{-\frac{n}{n-2}} \overline{\mathrm{D}^{\mathbf{S}}\left(f_{1}^{-\frac{1}{n-2}} \psi\right)} .
$$

Note that the volume forms of the boundary $\partial \mathrm{M}$ in the metric $g$ and $\bar{g}=f_{1}^{\frac{4}{n-2}} g$ are related by the formula

$$
d s(\bar{g})=f_{1}^{\frac{2(n-1)}{n-2}} d s(g)
$$


and then the boundary term is given by

$$
\begin{aligned}
\int_{\partial \mathrm{M}}\left\langle\overline{\mathrm{D}}^{\mathrm{S}}(\bar{\varphi}), \bar{\varphi}\right\rangle d s(\bar{g}) & =\int_{\partial \mathrm{M}}\left\langle\mathrm{D}^{\mathrm{S}}\left(f_{1}^{-\frac{1}{n-2}} \psi\right), f_{1}^{-\frac{1}{n-2}} \psi\right\rangle d s(g) \\
& =\int_{\partial \mathrm{M}}\left\langle\gamma\left(d\left(f_{1}^{-\frac{1}{n-2}}\right)\right) \psi, f_{1}^{-\frac{1}{n-2}} \psi\right\rangle d s(g)+\int_{\partial \mathrm{M}} f_{1}^{-\frac{2}{n-2}}\left\langle\mathrm{D}^{\mathrm{S}} \psi, \psi\right\rangle d s(g) .
\end{aligned}
$$

We pointed out that the first term of the preceding identity is purely imaginary, so inequality (20) gives

$$
\int_{\mathrm{M}}\left(\frac{1}{4} \mu_{1}(\mathrm{~L})-\frac{n-1}{n}\left|\lambda_{k}^{\mathrm{CHI}}\right|^{2}\right)|\bar{\varphi}|^{2} f_{1}^{-\frac{4}{n-2}} d v(\bar{g}) \leq \int_{\partial \mathrm{M}} f_{1}^{-\frac{2}{n-2}}\left\langle\mathrm{D}^{\mathbf{S}} \psi, \psi\right\rangle d s(g) .
$$

Using the fact that the spinor $\psi$ satisfies the eigenvalue boundary problem (16) and that $\mathrm{GD}^{\mathrm{S}}=\mathrm{D}^{\mathrm{S}} \mathrm{G}$ (using property (15)), we obtain:

$$
\left\langle\mathrm{D}^{\mathbf{S}} \psi, \psi\right\rangle=\left\langle\gamma(\nu) \mathrm{G}\left(\mathrm{D}^{\mathbf{S}} \psi\right), \gamma(\nu) \mathrm{G} \psi\right\rangle=-\left\langle\mathrm{D}^{\mathbf{S}} \psi, \psi\right\rangle
$$

hence, the desired inequality follows. Assume now that equality is achieved. So equality occurs in (18) and then by Proposition 2, the spinor field $\bar{\varphi}$ satisfies the following equation

$$
\overline{\mathrm{P}}_{X}(\bar{\varphi})=\bar{\nabla}_{X} \bar{\varphi}+\frac{1}{n} \bar{\gamma}(X) \overline{\mathrm{D}}(\bar{\varphi})=0, \quad \forall X \in \Gamma(\mathrm{TM}) .
$$

Moreover, using equation (17), we conclude that this spinor field satisfies the generalized Killing equation

$$
\bar{\nabla}_{X} \bar{\varphi}+\frac{\lambda}{n} f_{1}^{-\frac{2}{n-2}} \bar{\gamma}(X) \bar{\varphi}=0
$$

for all $X \in \Gamma(\mathrm{TM})$ and where $\frac{\lambda}{n} f_{1}^{-\frac{2}{n-2}}$ is a real-valued function. However, it is a wellknown fact (see Hij86) that, because $f_{1}$ is a real-valued function, $f_{1}$ is constant. Then the spinor field $\varphi$ (and so the spinor field $\psi$ ) is a real Killing spinor, i.e. it satisfies the Killing equation

$$
\nabla_{X} \psi+\frac{c}{2} \gamma(X) \psi=0, \quad \forall X \in \Gamma(\mathrm{TM}),
$$

where $c$ is a positive real number given by $c=\frac{2 \lambda}{n}$. Moreover this implies that the length $|\psi|^{2}$ is a non-zero constant and that $(\mathrm{M}, g)$ is an Einstein manifold with Ricci curvature Ric $=(n-1) c^{2} g$ whose boundary is minimal. Now consider the function given by $\mathrm{F}=\langle\mathrm{G}(\psi), \psi\rangle$ which is real-valued because the chirality operator $\mathrm{G}$ is pointwise selfadjoint. We then check that $\mathrm{F}$ is non identically zero on $\mathrm{M}$ since using Formula (8), we have

$$
n c \int_{\mathrm{M}} \mathrm{F} d v(\bar{g})=\int_{\partial \mathrm{M}}|\psi|^{2} d s(\bar{g}),
$$

and then, as $\psi$ is a non-zero constant lenght spinor field, we obtain $c>0$ and $\mathrm{F} \not \equiv 0$. We now prove that the function $\mathrm{F}$ satisfies the boundary problem

$$
\begin{cases}\Delta \mathrm{F}=n c^{2} \mathrm{~F} & \text { on } \mathrm{M} \\ \mathrm{F}_{\mid \partial \mathrm{M}}=0 & \text { along } \partial \mathrm{M} .\end{cases}
$$


An easy calculation using the Killing equation gives $\Delta \mathrm{F}=n c^{2} \mathrm{~F}$ on $\mathrm{M}$. The spinor field $\psi$ satisfies the eigenvalue boundary problem (16), in particular we have

$$
\gamma(\nu) \mathrm{G} \psi_{\mid \partial \mathrm{M}}=\mp \psi_{\mid \partial \mathrm{M}}
$$

and then

$$
\mathrm{F}_{\mid \partial \mathrm{M}}=\left\langle\mathrm{G} \psi_{\mid \partial \mathrm{M}}, \psi_{\mid \partial \mathrm{M}}\right\rangle= \pm\left\langle\gamma(\nu) \psi_{\mid \partial \mathrm{M}}, \psi_{\mid \partial \mathrm{M}}\right\rangle,
$$

hence $\mathrm{F}_{\mid \partial \mathrm{M}}=0$ since the right hand side of the last equation is purely imaginary. Applying the Obata Theorem for manifold with boundary proved by Reilly in Rei77] allows to conclude that $\mathrm{M}$ is conformally equivalent to $\mathbb{S}_{+}^{n}(r)$ with $r=\frac{1}{c}$.

q.e.d.

The proof of Theorem 5 is based on a well-chosen conformal metric $\bar{g}=f^{\frac{4}{n-2}} g$ which has no sense if $n=2$. However, we can apply with slight modifications, the argument used in Bär92 for the case of compact surfaces without boundary.

Theorem 6. Let $\left(\mathrm{M}^{2}, g\right)$ be a compact Riemannian spin surface of genus 0 . Suppose $\partial \mathrm{M}$ is compact connected. Under the CHI boundary condition, the spectrum of the Dirac operator $\mathrm{D}$ of $\mathrm{M}$ is a non-decreasing sequence of real numbers $\left\{\lambda_{k}^{\mathrm{CHI}} / k \in \mathbb{Z}\right\}$ which satisfies

$$
\left(\lambda_{k}^{\mathrm{CHI}}\right)^{2} \geq \frac{2 \pi}{\operatorname{Area}\left(\mathrm{M}^{2}, g\right)} .
$$

Equality holds for the smallest eigenvalue if and only if $\mathrm{M}^{2}$ is isometric to a standard hemisphere $\mathbb{S}_{+}^{2}(r)$ with $r=\frac{1}{\lambda_{ \pm 1}^{\mathrm{CHI}}}$.

Proof: We first show that under this boundary condition, any eigenvalue $\lambda^{\mathrm{CHI}}$ of the Dirac operator satisfies

$$
\left(\lambda^{\mathrm{CHI}}\right)^{2} \geq \frac{1}{2} \sup _{u} \inf _{\mathrm{M}}\left(\overline{\mathrm{R}} e^{2 u}\right)
$$

where the supremum is taken over all the functions $u$ satisfying $\frac{\partial u}{\partial \nu}+\mathrm{H}=0$, where $\mathrm{R}$ (resp. $\bar{R}$ ) is the scalar curvature of $g$ (resp. $\bar{g}=e^{2 u} g$ ) and $\mathrm{H}$ is the geodesic curvature of $\partial \mathrm{M}$. So for a metric $\bar{g}=e^{2 u} g$ with $\frac{\partial u}{\partial \nu}+\mathrm{H}=0$, in the conformal class of $g$, the associated Dirac operators are related by the following identity

$$
\overline{\mathrm{D}}\left(e^{-\frac{1}{2} u} \bar{\psi}\right)=e^{-\frac{3}{2} u} \overline{\mathrm{D} \psi} \text {. }
$$

Using the argument given in the proof of Theorem 5 and the fact that $\bar{H}=0$ for all $u$ such that $\frac{\partial u}{\partial \nu}+\mathrm{H}=0$ lead to the inequality

$$
\int_{\mathrm{M}}\left(\frac{\bar{R}}{4} e^{2 u}-\frac{\left|\lambda_{k}^{\mathrm{MIT}}\right|^{2}}{2}\right) e^{-2 u}|\bar{\varphi}|^{2} d v(\bar{g}) \leq \int_{\partial \mathrm{M}}\left\langle\overline{\mathrm{D}^{\mathbf{S}}}(\bar{\varphi}), \bar{\varphi}\right\rangle d s(\bar{g}),
$$

where $\varphi=e^{-\frac{1}{2} u} \psi$ and $\psi \in \Gamma(\Sigma \mathrm{M})$ satisfies the boundary problem (16). The conformal covariance of the CHI boundary condition gives (23). We now give an explicit calculation 
of the right-hand side of Inequality (23) . First, remark that for $n=2$, the transformations of the scalar curvature and the geodesic curvature give

$$
\left\{\begin{array}{l}
\overline{\mathrm{R}} e^{2 u}=\mathrm{R}+2 \Delta u \\
\bar{H} e^{u}=\frac{\partial u}{\partial \nu}+\mathrm{H}
\end{array}\right.
$$

and then, by assumption on the function $u, \overline{\mathrm{H}}=0$. Now, note that for such a function $u$

$$
\begin{aligned}
\inf _{\mathrm{M}}\left(\overline{\mathrm{R}} e^{2 u}\right) & \leq \frac{1}{\operatorname{Area}\left(\mathrm{M}^{2}, g\right)}\left(\int_{\mathrm{M}} \overline{\mathrm{R}} e^{2 u} d v(g)+2 \int_{\partial \mathrm{M}} \overline{\mathrm{H}} d s(g)\right) \\
& \leq \frac{1}{\operatorname{Area}\left(\mathrm{M}^{2}, g\right)}\left(\int_{\mathrm{M}} \operatorname{R} d v(g)+2 \int_{\partial \mathrm{M}} \mathrm{H} d s(g)\right)=\frac{4 \pi \chi\left(\mathrm{M}^{2}\right)}{\operatorname{Area}\left(\mathrm{M}^{2}, g\right)}
\end{aligned}
$$

by Stokes and Gauss-Bonnet Formula for surfaces with boundary (see [DC91]). Let $u_{1}$ a solution of the boundary problem (see Tay96 for example)

$$
\begin{cases}2 \Delta u_{1}=\frac{1}{\operatorname{Area}\left(\mathrm{M}^{2}, g\right)}\left(\int_{\mathrm{M}} \mathrm{R} d v(g)+2 \int_{\partial \mathrm{M}} \mathrm{H} d s(g)\right)-\mathrm{R} & \text { on } \mathrm{M} \\ \frac{\partial u_{1}}{\partial \nu}+\mathrm{H}=0 & \text { along } \partial \mathrm{M},\end{cases}
$$

then an easy calculation gives

$$
\inf _{\mathrm{M}}\left(\overline{\mathrm{R}} e^{2 u_{1}}\right)=\frac{4 \pi \chi\left(\mathrm{M}^{2}\right)}{\text { Area }\left(\mathrm{M}^{2}, g\right)} .
$$

From Inequality (23), we obtain:

$$
\left(\lambda_{k}^{\mathrm{CHI}}\right)^{2} \geq \frac{2 \pi \chi\left(\mathrm{M}^{2}\right)}{\operatorname{Area}\left(\mathrm{M}^{2}, g\right)} .
$$

However, the surface $\mathrm{M}$ is of genus 0 and its boundary has one connected component, then $\chi(\mathrm{M})=1$ and so Inequality (22) follows immediately. The equality case is treated as in the proof of Theorem 5 .

q.e.d.

Remark 2. The Euler-Poincaré characteristic of a surface of genus $g \geq 0$ and with $m \geq 1$ boundary components is given by (see [Hir76])

$$
\chi(\mathrm{M})=2-2 g-m \text {. }
$$

It is a simple fact that

$$
\chi(\mathrm{M})>0 \Leftrightarrow \chi(\mathrm{M})=1 \Leftrightarrow(g=0 \quad \text { et } \quad m=1) .
$$

We can now relate the Yamabe invariant of the manifold $M$ with the eigenvalues of the Dirac operator under the boundary condition associated with a chirality operator. This conformal invariant, denoted by $\mu(\mathrm{M})$, has been introduced by Escobar in Esc92 in order to solve the Yamabe problem for manifolds with boundary and has the following variational characterization

$$
\mu(\mathrm{M})=\inf _{u \in C^{1}(\mathrm{M}), u \neq 0} \frac{\int_{\mathrm{M}}\left(\frac{2}{n-2}|\nabla u|^{2}+\frac{1}{2(n-1)} \mathrm{R} u^{2}\right) d v(g)+\int_{\partial \mathrm{M}} \mathrm{H} u^{2} d s(g)}{\left(\int_{\mathrm{M}} u^{\frac{2 n}{n-2}} d s(g)\right)^{\frac{n-2}{n}}} .
$$


He proved that $\mu(\mathrm{M})$ has the same sign as $\mu_{1}(\mathrm{~L})$ and it is invariant with respect to conformal changes of the metric on M. The Hölder inequality applied to an eigenfunction $f_{1}$ associated with $\mu_{1}(\mathrm{M})$ gives

$$
\mu_{1}(\mathrm{~L}) \geq \frac{\mu(\mathrm{M})}{\operatorname{vol}(\mathrm{M}, g)^{\frac{2}{n}}}
$$

and equality implies that $f_{1}$ is constant. Thus, from Theorem 5 and Theorem 6, we have:

Corollary 7. Let $\left(\mathrm{M}^{n}, g\right)$ be an n-dimensional compact Riemannian spin manifold with non-empty compact connected boundary $\partial \mathrm{M}$ and $n \geq 2$. Under the $\mathrm{CHI}$ boundary condition, any eigenvalue $\lambda^{\mathrm{CHI}}$ of the Dirac operator D satisfies

$$
\left|\lambda^{\mathrm{CHI}}\right|^{2} \operatorname{vol}(\mathrm{M}, g)^{\frac{2}{n}} \geq \frac{n}{4(n-1)} \mu(\mathrm{M}) \text {. }
$$

Remark 3. Theorem [5 and Theorem [6 can also be proved for the MIT bag boundary condition used in [HMR02] and [HMZ02 for example. This condition is defined as being the orthogonal projection on the eigensubbundles of the pointwise endomorphism $i \gamma(\nu)$ acting on $\mathbf{S}(\partial \mathrm{M})$ associated with the eigenvalues \pm 1 , i.e

$$
\begin{aligned}
\mathbb{B}_{\mathrm{MIT}}^{ \pm}: \mathrm{L}^{2}(\mathbf{S}(\partial \mathrm{M})) & \longrightarrow \mathrm{L}^{2}\left(\mathrm{~V}^{ \pm}\right) \\
\varphi & \longmapsto \frac{1}{2}(\mathrm{Id} \pm i \gamma(\nu)) \varphi .
\end{aligned}
$$

This differential operator satisfies the Lopatinsky-Shapiro ellipticity conditions, and so it defines a local elliptic boundary condition for the Dirac operator of the manifold $\mathrm{M}$. We can then show (see [HMR02 ) that under the $\mathbb{B}_{\text {MIT }}^{-}$(resp. $\mathbb{B}_{\text {MIT }}^{+}$) condition, the spectrum of the Dirac operator is a discrete set of complex numbers with positive (resp. negative) imaginary part. Furthemore, we can show that any eigenvalue $\lambda^{\mathrm{MIT}}$ under this boundary condition satifies for $n \geq 3$

$$
\left|\lambda^{\mathrm{MIT}}\right|^{2}>\frac{n}{4(n-1)} \mu_{1}(\mathrm{~L})
$$

and for $n=2$

$$
\left|\lambda^{\mathrm{MIT}}\right|^{2}>\frac{2 \pi}{\operatorname{Area}\left(\mathrm{M}^{2}, g\right)}
$$

Equality cannot be achieved in (25) and (26) otherwise there should exist on the manifold $\mathrm{M}$ a generalized Killing spinor with imaginary Killing function and then the scalar curvature should be non-positive. However, if equality holds, the scalar curvature of M is positive and so there is a contradiction.

\section{REFERENCES}

[Bär92] C. Bär, Lower eigenvalue estimate for Dirac operator, Math. Ann. 293 (1992), 39-46.

[Bär98] _ Extrinsic bounds of the Dirac operator, Ann. Glob. Anal. Geom. 16 (1998), 573-596.

[Bau89] H. Baum, Complete Riemannian manifolds with imaginary Killing spinors, Ann. Glob. Anal. Geom. 7 (1989), 205-226.

[BBW93] B. Booß-Bavnbek and K. P. Wojciechowski, Elliptic boundary problems for the Dirac operator, Birkhäuser, Basel, 1993.

[DC91] M. Do Carmo, Differential forms and applications, Springer-Verlag, 1991.

[DW95] S. Dürr and A. Wipf, Gauge theories in a bag, Nucl. Phys. B 443 (1995), 201-232. 
[Esc92] J. F. Escobar, The Yamabe problem on manifolds with boundary, J. Diff. Geom. 35 (1992), $21-84$.

[Fri80] T. Friedrich, Der erste Eigenwert des Dirac-Operators einer kompakten Riemannschen Mannigfaltigkeit nicht negativer Skalarkrümmung, Math. Nach. 97 (1980), 117-146.

[Fri00] _ Dirac operators in Riemannian geometry, vol. 25, Amer. Math. Soc. Graduate Studies in Math., 2000.

[Hij86] O. Hijazi, A conformal lower bound for the smallest eigenvalue of the Dirac operator and Killing spinors, Commun. Math. Phys. 25 (1986), 151-162.

[Hij91] , Première valeur propre de l'opérateur de Dirac et nombre de Yamabe, C. R. Acad. Sci. Paris 313 (1991), 865-868.

[Hir76] M. W. Hirsch, Differential Topology, New York: Springer-Verlag, 1976.

[Hit74] N. Hitchin, Harmonic spinors, Adv. Math. 14 (1974), 1-55.

[HMR02] O. Hijazi, S. Montiel, and S. Roldán, Eigenvalue boundary problems for the Dirac operator, Commun. Math. Phys. 231 (2002), 375-390.

[HMZ01] O. Hijazi, S. Montiel, and X. Zhang, Dirac operator on embedded hypersurfaces, Math. Res. Lett. 8 (2001), 20-36.

[HMZ02] _ Conformal lower bounds for the Dirac operator on embedded hypersurfaces, Asian J. Math. 6 (2002), 23-36.

[Hör85] L. Hörmander, The analysis of linear partial differential operators III, Springer, Berlin, 1985.

[LM89] H. B. Lawson and M. L. Michelsohn, Spin Geometry, Princeton University Press ed., vol. 38, Princeton Math. Series, 1989.

[Lop53] Ya. B. Lopatinskiú, On a method for reducing boundary problems for a system of differential equations of elliptic type to regular integral equations, Ukrain. Math. Ž. 5 (1953), 123-151, (Russian).

[Mor01] B. Morel, Eigenvalue estimates for the Dirac-Schrödinger operators, Journal of Geometry and Physics 38 (2001), 1-18.

[Rei77] R. C. Reilly, Application of the Hessian operator in a Riemannian manifold, Indiana Univ. Math. J. 26 (1977), 459-472.

[Tay96] M. Taylor, Partial differentiel equations, Vol. 1: Basic theory, Springer, 1996.

[Tra95] A. Trautman, The Dirac operator on hypersurfaces, Acta Phys. Polon. 6 (1995), 1283-1310.

[Yam60] H. Yamabe, On a deformation of Riemannian structures on compact manifolds, Osaka Math. J. (1960), 21-37.

Author address:

Simon Raulot,

Institut Élie Cartan BP 239

Université de Nancy 1

54506 Vandœuvre-lès -Nancy Cedex

France

E-Mail: raulot@iecn.u-nancy.fr 